\title{
The complex geometry of the spherical pendulum
}

Frits Beukers and Richard Cushman

Dedicated to Don Saari on his sixtieth birthday.

Abstract. We show that the complexified spherical pendulum has monodromy.

\section{Introduction}

In this paper we describe the geometry of the energy momentum mapping of the complexified spherical pendulum. For background on the classical spherical pendulum we refer the reader to [4 5 chpt. IV]. We show that this complex Hamiltonian system is Mumford-Jacobi completely integrable $[9 \Gamma$ p.3.51-3.53] and admits an analogue of the period lattice $[5]$ and action angle coordinates [4 4 Apend. D]. Following the variation of the period lattice around a closed loop in the complement of the discriminant locus of a family of elliptic curves (see equation (9) below) Twe show that the complexified spherical pendulum has monodromy [4 Гр.383].

The monodromy of the complexified spherical pendulum also has been described in recent papers by Audin [2] (unpublished) and Gavrilov and Vivolo [6]. Audin starts with a Lax pair related to the complexified spherical pendulum. She then studies the geometry of a singular normalization $\widetilde{C}_{c}$ of the family of affine elliptic curves $C_{c}$ :

$$
\mu^{2}+\lambda^{4}+2 c_{2} \lambda^{3}+2 c_{1} \lambda^{2}+1=0
$$

2000 Mathematics Subject Classification. Primary 58Z05; Secondary 70H06.

Key words and phrases. Liouville integrable system, complex spherical pendulum, monodromy.

The work of the second author was (partially) supported by European Commission funding for the Research Training Network "Mechanics and Symmetry in Europe" (MASIE), Contract No. HPRN-CT-2000-00113. 
which is part of the spectral curve of the Lax pair. Using PicardLefschetz techniques $\Gamma$ she then computes the variation of the period map $\mathrm{H}_{1}\left(\tilde{C}_{c}, \mathbf{Z}\right) \rightarrow \mathbf{C}^{2}$ as $c=\left(c_{1}, c_{2}\right)$ traces out a curve in the complement of the discriminant locus of $C_{c}$.

The paper of Gavrilov and Vivolo starts with the discriminant locus of $C_{c}$ and then constructs a set of action-angle coordinates $\Gamma$ which gives a simple proof of Horozov's theorem for the spherical pendulum. Using these action-angle coordinates and Picard-Lefschetz theory Tthey show that the complex spherical pendulum has monodromy.

There are serveral difficulties with the approaches to monodromy used in the above papers. The most serious one is that the exact relation of the family $C_{c}$ with the dynamics of the complexified spherical pendulum is no where explained. Audin's choice of the singular normalization $\widetilde{C}_{c}$ of the family $C_{c}$ is not motivated from the dynamics. Also she does not explain what the generalized Jacobian for $\widetilde{C}_{c}$ is. (The published literature on generalized Jacobians is inaccessible to dynamicists).

On the other hand Twe think that our explanation of monodromy is direct. The key obsevation is that a level set of a regular value of the energy momentum map is a $\mathbf{C}^{*}$-bundle over an elliptic curve. Moreover $\Gamma$ it is a quotient of $\mathrm{C}^{2}$ by a rank 3 lattice. The inverse of the quotient map is simply given by the action angle variables on the level set. We note that the monodromy we find is not the same the same as the classic Picard-Fuchs monodromy described in [3Г p.657-693] Г because it involves a differential form of the third kind $\mathrm{Tnor}$ it is the same as the monodromy given in $[\mathbf{1}]$. We have just given an analytic proof of a geometric explanation of monodromy suggested by Knörrer [8].

\section{The complexified spherical pendulum}

In this section we describe the complex Hamiltonian system given by the complexified spherical pendulum.

Let $\langle$,$\rangle be the standard symmetric bilinear form on \mathbf{C}^{3} \Gamma$ namely $\Gamma$ $\langle x, y\rangle=\sum_{i=1}^{3} x_{i} y_{i}$ for $x, y \in \mathbf{C}^{3}$. Set $Q=\left\{x \in \mathbf{C}^{3} \mid\langle x, x\rangle=1\right\}$. Then $Q$ is the complexification of the 2-sphere $S^{2}$ in $\mathbf{R}^{3}$. Let

$$
M=T Q=\left\{(x, v) \in Q \times \mathbf{C}^{3} \mid\langle x, v\rangle=0\right\}
$$

be the tangent bundle of $Q$ with bundle projection

$$
\text { pr }: M \rightarrow Q:(x, v) \rightarrow x .
$$


On $M$ there is a holomorphic nondegenerate closed 2-form $\omega$ which is obtained by pulling back the canonical symplectic form on the cotangent bundle of $Q$ by the flat map associated to $\langle$,$\rangle . Note that M$ is a smooth subvariety of $\mathbf{C}^{3} \times \mathbf{C}^{3}$.

Recall [4 4 p. 149] that as a constrained Hamiltonian system $(H, M, \omega) \Gamma$ the complexified spherical pendulum has Hamiltonian

$$
H: M \rightarrow \mathbf{C}:(x, v) \rightarrow \frac{1}{2}\langle v, v\rangle+x_{3} .
$$

Moreover $\Gamma i t s$ motion is governed by the Hamiltonian vector field $X_{H} \Gamma$ whose integral curves satisfy

$$
\begin{aligned}
\dot{x} & =v \\
\dot{v} & =-e_{3}+\left(\left\langle x, e_{3}\right\rangle-\langle v, v\rangle\right) x .
\end{aligned}
$$

The group

$$
\mathrm{SO}(2, \mathbf{C})=\left\{A=\left(\begin{array}{rr}
a & -b \\
b & a
\end{array}\right) \in \mathrm{Gl}(2, \mathbf{C}) \mid a^{2}+b^{2}=1\right\}
$$

acts on $M$ by

$$
\begin{aligned}
& \widetilde{\Phi}: \mathrm{SO}(2, \mathrm{C}) \times M \rightarrow M: \\
& \quad(A,(x, v)) \rightarrow\left(\left(\begin{array}{rr}
A & 0 \\
0 & 1
\end{array}\right) x,\left(\begin{array}{rr}
A & 0 \\
0 & 1
\end{array}\right) v\right) .
\end{aligned}
$$

This action is HamiltonianThas momentum mapping

$$
L: M \rightarrow \mathbf{C}:(x, v) \rightarrow x_{1} v_{2}-x_{2} v_{1},
$$

and has an associated Hamiltonian vector field $X_{L}$ whose integral curves satisfy

$$
\begin{aligned}
& \dot{x}=e_{3} \times x \\
& \dot{v}=e_{3} \times v .
\end{aligned}
$$

Since $\widetilde{\Phi}$ leaves $H$ invariant $\Gamma L$ is an integral of $X_{H}$. Let

$$
\widehat{\mathcal{E M}}: M \rightarrow \mathbf{C}^{2}:(x, v) \rightarrow(H(x, v), L(x, v))
$$

be the energy momentum mapping.

For later purposes it is convenient to introduce new coordinates $\left(\xi_{1}, \xi_{2}, z, \eta_{1}, \eta_{2}, y\right)$ on $\mathbf{C}^{3} \times \mathbf{C}^{3}$ where

$$
\xi_{1}=x_{1}+i x_{2}, \xi_{2}=x_{1}-i x_{2}, \eta_{1}=v_{1}+i v_{2}, \eta_{2}=v_{1}-i v_{2} .
$$

In these coordinates the defining equations of $M$ are

$$
\xi_{1} \xi_{2}+1=1-z^{2} \text { and } \xi_{1} \eta_{2}+\xi_{2} \eta_{1}+2 z y=0 .
$$

The energy momentum mapping $\widehat{\mathcal{E M}}$ becomes

$$
\mathcal{E M}: M \rightarrow \mathbf{C}^{2}:(\xi, z, \eta, y) \rightarrow\left(c_{1}, c_{2}\right),
$$


where

$$
c_{1}=\frac{1}{2}\left(\eta_{1} \eta_{2}+y^{2}\right)+z \text { and } c_{2}=\frac{1}{i}\left(\xi_{2} \eta_{1}-\xi_{1} \eta_{2}\right) .
$$

In addition $\Gamma$ the $\mathrm{SO}(2, \mathbf{C})$-action $\tilde{\Phi}$ becomes the $\mathbf{C}^{*}$-action

$$
\begin{aligned}
\Phi: & \mathbf{C}^{*} \times M \rightarrow M: \\
& \left(\lambda,\left(\xi_{1}, \xi_{2}, z, \eta_{1}, \eta_{2}, y\right)\right) \rightarrow\left(\lambda \xi_{1}, \lambda^{-1} \xi_{2}, z, \lambda \eta_{1}, \lambda^{-1} \eta_{2}, z\right) .
\end{aligned}
$$

We have

Proposition 2.1. Let $M^{\circ}$ be the subset of $M$ consisting of all points $\left(\xi_{1}, \xi_{2}, z, \eta_{1}, \eta_{2}, y\right)$ where at least one of the monomials $\xi_{1} \xi_{2}, \xi_{1} \eta_{2}$, $\xi_{2} \eta_{1}, \eta_{1} \eta_{2}$ is nonzero. Then the following statements hold.

1. The $\mathbf{C}^{*}$-action $\Phi$ on $M^{\circ}$ is free and proper.

2. The map

$$
\pi: M^{\circ} \rightarrow \mathbf{C}^{4}:\left(\xi_{1}, \xi_{2}, z, \eta_{1}, \eta_{2}, y\right) \rightarrow\left(z, y, c_{1}, c_{2}\right)
$$

is the quotient map $M^{\circ} \rightarrow M^{\circ} / \mathbf{C}^{*}$ of the $\mathbf{C}^{*}$-action $\Phi$.

3. The image of the map $\pi$ is the subvariety $E^{\circ}$ of $\mathrm{C}^{4}$ defined by

$$
\left\{\left(z, y, c_{1}, c_{2}\right) \in \mathbf{C}^{4} \mid y^{2}=f_{c}(z)\right\} \backslash( \pm 1,0, \pm 1,0),
$$

where

$$
f_{c}(z)=2\left(z^{2}-1\right)\left(z-c_{1}\right)-c_{2}^{2}
$$

4. The map $\pi: M^{\circ} \rightarrow E^{\circ}$ is the projection map of a $\mathbf{C}^{*}$-bundle over $E^{\circ}$.

Proof. 1. First of all note that the map $\Phi_{\lambda}$ with $\lambda \neq 1$ has a fixed point if and only if $\xi_{1}=\xi_{2}=\eta_{1}=\eta_{2}=0$. Hence $\mathbf{C}^{*}$ acts freely on $M^{\circ}$. To verify that the action $\Phi$ is proper $\Gamma i t$ suffices to show that for any two compact subsets $K_{1}$ and $K_{2}$ of $M^{\circ}$ the set $L=\left\{\lambda \in \mathbf{C}^{*} \mid K_{1} \cap\right.$ $\left.\Phi_{\lambda}\left(K_{2}\right) \neq \emptyset\right\}$ is compact. Since $K_{2} \subseteq M^{\circ}$ is compact $\Gamma$ at least one of the products $\left|\xi_{1} \xi_{2}\right|,\left|\xi_{1} \eta_{2}\right|,\left|\xi_{2} \eta_{1}\right| \Gamma\left|\eta_{1} \eta_{2}\right|$ restricted to $K_{2}$ is bounded below by a positve number. Suppose for example that $\left|\xi_{1} \xi_{2}\right|$ is bounded below by $\rho>0$. In addition $\Gamma$ the modulus of coordinates of points in $K_{1}$ are bounded above by some number $R$. Hence for every $\lambda \in L$ there is a point in $K_{2}$ whose $\xi_{1}$ and $\xi_{2}$ coordinates satisfy $|\lambda|\left|\xi_{1}\right|<R$ and $|\lambda|^{-1}\left|\xi_{2}\right|<R$. Using the upper and lower bounds for $\left|\xi_{1}\right|$ and $\left|\xi_{2}\right|$ we find that $\rho / R^{2}<|\lambda|<R^{2} / \rho$. Thus $L \subseteq \mathrm{C}$ is contained in a bounded set which is bounded away from zero. A standard argument using the continuity of $\Phi$ shows that $L$ is closed. Consequently $L$ is compact. 
2. Given the equation of $M$ and the definition of $c_{1}, c_{2}$ T the following equalities hold for points of $M^{\circ}$

$$
\begin{aligned}
& \xi_{1} \xi_{2}=1-z^{2} \\
& \xi_{1} \eta_{2}=-z y-i c_{2} \\
& \xi_{2} \eta_{1}=-z y+i c_{2} \\
& \eta_{1} \eta_{2}=2\left(c_{1}-z\right)-y^{2} .
\end{aligned}
$$

Note that the points of $M^{\circ}$ have the property that at least one of the right hand sides of the equations ( 8 ) is nonzero. Conversely choose $p=\left(z, y, c_{1}, c_{2}\right)$ so that at least one of the right hand sides of (8) is nonzero Wwe see that the inverse image of $p$ under the map $\pi$ is a single $\mathbf{C}^{*}$-orbit. Hence $\pi$ is the quotient map of the action $\Phi$.

3. Note that the identity $\left(\xi_{1} \xi_{2}\right)\left(\eta_{1} \eta_{2}\right)=\left(\xi_{1} \eta_{2}\right)\left(\xi_{2} \eta_{1}\right)$ gives rise to

$$
\left(1-z^{2}\right)\left(2\left(c_{1}-z\right)-y^{2}\right)=-\left(z y+i c_{2}\right)\left(-z y+i c_{2}\right),
$$

which can be simplified to

$$
y^{2}=f_{c}(z)=2\left(z^{2}-1\right)\left(z-c_{1}\right)-c_{2}^{2} .
$$

4. Finally if $\mathbf{C}^{*}$ acts freely and properly on a complex manifold $V$ Then the quotient map $V \rightarrow V / \mathbf{C}^{*}$ is the projection map of a $\mathbf{C}^{*}$-bundle over $V / \mathbf{C}^{*}$. In particular $\Gamma$ this holds when $V=M^{\circ}$.

For any value of $c=\left(c_{1}, c_{2}\right)$ consider the affine elliptic curve

$$
E_{c}: y^{2}=f_{c}(z) \text {. }
$$

It is easy to check that $E_{c}$ is smooth if and only if $f_{c}$ has no multiple roots $\Gamma$ that is $\Gamma$ if and only if $c$ does not lie on the discriminant locus $\Delta$ defined by

$$
0=\operatorname{g.c.d}\left(f_{c}, f_{c}^{\prime}\right)=\frac{27}{4} c_{2}^{4}+2 c_{1}\left(c_{1}^{2}-9\right) c_{2}^{2}-4\left(c_{1}^{2}-1\right)^{2} .^{\dagger}
$$

The quotient variety $E^{\circ}=M^{\circ} / \mathrm{C}^{*}$ can be considered as a family of affine elliptic curves $E_{c}$ parametrized by $c=\left(c_{1}, c_{2}\right)$.

Let

$$
E^{n s}=\left\{\left(c_{1}, c_{2}, z, y\right) \in\left(\mathbf{C}^{2} \backslash \Delta\right) \times \mathbf{C}^{2} \mid y^{2}=f_{c}(z)\right\} .
$$

Then $E^{n s}$ is the union of all smooth affine elliptic curves $E_{c}$. Since the points $( \pm 1,0)$ lie in $\Delta$ Tit follows that $E^{n s} \subseteq E^{\circ}$. Let $M^{n s}$ be the inverse image of $E^{n s}$ under the quotient map $\pi$ and let $M_{c}$ be the inverse image of $E_{c}$ under $\pi$. Then $M_{c}$ is the $c$-level set of the energy

\footnotetext{
${ }^{\dagger}$ Since $c_{1}=\frac{3}{2} s-\frac{1}{2} \frac{1}{s}, c_{2}^{2}=-\frac{1}{s}\left(1-s^{2}\right), s \in \mathrm{C}^{*}$ is a parametrization of $\Delta$ by rational functions, it follows that the genus its projectivization $\bar{\Delta}$ is 0 . Because $\bar{\Delta}$ has three ordinary double points, it is an immersion of a 2 -sphere in complex projective 2-space with three normal crossings.
} 
momentum map $\mathcal{E M}$. The geometrical structure of these level sets will become a major issue later on in this paper. By proposition 2.1 we know that $\pi: M^{n s} \rightarrow E^{n s}$ is a $\mathbf{C}^{*}$-bundle. If we restrict this bundle to the nonsingular curve $E_{c}$ एwe see that $M_{c}$ is a $\mathbf{C}^{*}$-bundle over $E_{c}$.

That the discriminant locus $\Delta$ plays an important role can be seen from the following proposition.

Proposition 2.2. The derivative of the map

$$
\mathcal{E} \mathcal{M} \mid M^{n s}: M^{n s} \rightarrow \mathrm{C}^{2} \backslash \Delta
$$

has maximal rank at every point of $M^{\text {ns }}$.

Proof. The map $\mathcal{E M} \mid M^{n s}$ is the composition of the projection map

$\pi: M^{n s} \rightarrow E^{n s}$ followed by the map

$$
\rho: E^{n s} \rightarrow \mathbf{C}^{2} \backslash \Delta:\left(z, y, c_{1}, c_{2}\right) \rightarrow\left(c_{1}, c_{2}\right) .
$$

Since $\pi$ is a quotient map of a free and proper action $\Gamma$ its derivative has maximal rank. Thus it suffices to show that the derivative of $\rho$ has maximal rank at every point of $E^{n s}$. Suppose not. Then there is a point $p$ of $E^{n s}$ where all the partial derivatives of $F\left(z, y, c_{1}, c_{2}\right)=-y^{2}+f_{c}(z)$ vanish and $y^{2}=f_{c}(z)$. Hence $y(p)=0$ and $\frac{\partial f_{c}(z)}{\partial z}(p)=0$. Moreover $\Gamma$ $f_{c}(z(p))=y^{2}(p)=0$. Thus $z(p)$ is a double zero of $f_{c}$ which is contrary to our assumption that $c \notin \Delta$.

\section{A partial compactification of $M_{c}$ and $E_{c}$}

In this section we find a partial compactification of the affine elliptic curve $E_{c}$ and the $c$-level set $M_{c}$ of the energy momentum mapping.

Since the curves $E_{c}$ in $E^{n s}$ are affine $\Gamma E_{c}$ is not complete. We would like to complete each $E_{c}$ by adding the point at infinity. We do this as follows. The variety $E^{n s}$ is an affine subvariety of $\left(\mathbf{C}^{2} \backslash \Delta\right) \times \mathbf{C}^{2}$ with coordinates $\left(\left(c_{1}, c_{2}\right),(z, y)\right)$ where $y^{2}=f_{c}(z)(7)$. We now embed the second factor $\mathbf{C}^{2}$ into the complex projective plane $\mathbf{P}^{2}$ by sending $(z, y)$ to the projective point $(z: y: 1)$. Denote the projective coordinates of $\mathbf{P}^{2}$ by $(z: y: u)$. Then $E^{n s}$ can be completed to the variety $\bar{E}^{n s}$ defined by

$\left\{\left(\left(c_{1}, c_{2}\right),(z: y: u)\right) \in\left(\mathbf{C}^{2} \backslash \Delta\right) \times \mathbf{P}^{2} \mid y^{2} u=2\left(z^{2}-u^{2}\right)\left(z-c_{1} u\right)-c_{2}^{2} u^{3}\right\}$.

For each value of $c=\left(c_{1}, c_{2}\right) \notin \Delta$ the curve $E_{c}$ has been completed by adding the point $\left(\left(c_{1}, c_{2}\right),(0: 1: 0)\right)$. We denote this completion by $\bar{E}_{c}$. Since $c \notin \Delta$ the curve $\bar{E}_{c}$ is a smooth compact 1-dimensional complex manifold and hence is an elliptic curve. 
We now complete the $\mathbf{C}^{*}$-bundle $\pi: M^{n s} \rightarrow E^{n s}$. Towards this goal $\Gamma$ we introduce the variety $\bar{M}^{n s} \subseteq\left(\mathbf{C}^{2} \backslash \Delta\right) \times \mathbf{P}^{6}$ with coordinates $\left(\left(c_{1}, c_{2}\right),\left(\xi_{1}: \xi_{2}: z: \eta_{1}: \eta_{2}: y: u\right)\right)$ defined by the equations

$$
\begin{aligned}
& \xi_{1} \xi_{2}=u^{2}-z^{2} \\
& \xi_{1} \eta_{2}=-z y-i c_{2} u^{2} \\
& \xi_{2} \eta_{1}=-z y+i c_{2} u^{2} \\
& \eta_{1} \eta_{2}=-y^{2}-2\left(c_{1} u-z\right) u
\end{aligned}
$$

and the condition that at least one of the monomials $\xi_{1} \xi_{2}, \xi_{1} \eta_{2}, \xi_{2} \eta_{1}, \eta_{1} \eta_{2}$ is nonzero. On $\bar{M}^{\text {ns }}$ we have a $\mathbf{C}^{*}$-action defined by

$$
\Psi: \mathrm{C}^{*} \times \bar{M}^{n s} \rightarrow \bar{M}^{n s}:
$$

$\left(\lambda,\left(\left(c_{1}, c_{2}\right),\left(\xi_{1}: \xi_{2}: z: \eta_{1}: \eta_{2}: y: u\right)\right)\right) \rightarrow\left(\left(c_{1}, c_{2}\right),\left(\lambda \xi_{1}: \lambda^{-1} \xi_{2}: z: \lambda \eta_{1}: \lambda^{-1} \eta_{2}: y: u\right)\right)$.

In a way similar to the proof of proposition 2.1 we can prove

Proposition 3.1. The map $\bar{\pi}: \bar{M}^{n s} \rightarrow \bar{E}^{n s}$ defined by

$$
\bar{\pi}\left(\left(c_{1}, c_{2}\right),\left(\xi_{1}: \xi_{2}: z: \eta_{1}: \eta_{2}: y: u\right)\right)=\left(\left(c_{1}, c_{2}\right),(z: y: u)\right)
$$

is the quotient map of the $\mathrm{C}^{*}$-action $\Psi$ on $\bar{M}^{\text {ns }}$. Since $\Psi$ is a free and proper $\mathbf{C}^{*}$-action, $\bar{\pi}$ is the projection map of a $\mathbf{C}^{*}$-bundle over $\bar{E}^{\text {ns }}$.

We easily check that $M^{n s}$ embeds in $\bar{M}^{n s}$ via

$$
\left(\left(c_{1}, c_{2}\right),\left(\xi_{1}, \xi_{2}, z, \eta_{1}, \eta_{2}, y\right)\right) \rightarrow\left(\left(c_{1}, c_{2}\right),\left(\xi_{1}: \xi_{2}: z: \eta_{1}: \eta_{2}: y: 1\right)\right) \text {. }
$$

The restriction of $\bar{\pi}$ to $M^{n s}$ is the projection map $\pi$ of proposition 2.1. The inverse image of $\bar{E}_{c}$ under $\bar{\pi}$ is denoted by $\bar{M}_{c}$. Thus $\bar{M}_{c}$ is a $\mathrm{C}^{*}$-bundle over the elliptic curve $\bar{E}_{c}$.

\section{Action angle coordinates}

In this section we obtain a complex analogue of the period lattice and action angle coordinates for the complex spherical pendulum.

We begin by choosing good coordinates on $M^{\text {ns }}$ in which to express the symplectic form $\omega$. Since $\omega$ is holomorphic on $M$ it is holomorphic on $M^{n s}$. Let $V$ be the Zariski open subset of $M^{n s}$ given by

$$
M^{n s} \cap\left\{\left(\xi_{1}, \xi_{2}, z, \eta_{1}, \eta_{2}, y\right) \in \mathbf{C}^{6} \mid \xi_{1} \xi_{2} \neq 0, \quad y \neq 0\right\} .
$$

At every point $p$ of $E^{n s}$ with $y \neq 0$ the variables $\left(\xi_{1}, z, c_{1}, c_{2}\right)$ can be used as local coordinates at $p$. At any point $p$ of $V$ we have $\xi_{1} \neq 0$. Supposing that the values of $\left(\xi_{1}, z, c_{1}, c_{2}\right)$ are known $\Gamma$ then the values of $\xi_{2}, \eta_{1}, \eta_{2}$ follow from

$$
\xi_{2}=\frac{\left(1-z^{2}\right)}{\xi_{1}}, \eta_{1}=\left(\frac{-z y+i c_{2}}{1-z^{2}}\right) \xi_{1}, \eta_{2}=\frac{-z y-i c_{2}}{\xi_{1}}
$$


by virtue of the defining equations of $M^{n s}$. Thus at every point of $V$ the variables $\left(\xi_{1}, z, c_{1}, c_{2}\right)$ can be used as local coordinates.

We now would like to write the symplectic form $\omega$ in terms of these coordinates.

Proposition 4.1. The symplectic form $\omega$ on $M$ restricted to $V$ is

$$
\frac{\mathrm{d} z}{y} \wedge \mathrm{d} c_{1}+i\left(\frac{z y-i c_{2}}{z^{2}-1} \frac{\mathrm{d} z}{y}-\frac{\mathrm{d} \xi_{1}}{\xi_{1}}\right) \wedge \mathrm{d} c_{2} .
$$

Proof. To see this note that the canonical symplectic form on $\mathbf{C}^{6}$ can be written as $\frac{1}{2}\left(\mathrm{~d} \xi_{1} \wedge d \eta_{2}+\mathrm{d} \xi_{2} \wedge \mathrm{d} \eta_{1}\right)+\mathrm{d} z \wedge \mathrm{d} y$. Since $\eta_{1}=\frac{-z y+i c_{2}}{\xi_{2}}$ and $\eta_{2}=-\frac{z y+i c_{2}}{\xi_{1}}$ on $V$ Twe obtain

$$
\mathrm{d} \eta_{1}=\frac{-z y+i c_{2}}{\xi_{2}^{2}} \mathrm{~d} \xi_{2}-\frac{1}{\xi_{2}} \mathrm{~d}(z y)+i \frac{1}{\xi_{2}} \mathrm{~d} c_{2}
$$

and

$$
\mathrm{d} \eta_{2}=\frac{z y+i c_{2}}{\xi_{1}^{2}} \mathrm{~d} \xi_{1}-\frac{1}{\xi_{1}} \mathrm{~d}(z y)-i \frac{1}{\xi_{1}} \mathrm{~d} c_{2}
$$

Hence

$$
\begin{aligned}
\left.\omega\right|_{V}=\mathrm{d} z & \wedge \mathrm{d} y-\frac{1}{2}\left(\frac{\mathrm{d} \xi_{1}}{\xi_{1}}+\frac{\mathrm{d} \xi_{2}}{\xi_{2}}\right) \wedge \mathrm{d}(z y) \\
& +\frac{1}{2 i}\left(\frac{\mathrm{d} \xi_{1}}{\xi_{1}}-\frac{\mathrm{d} \xi_{2}}{\xi_{2}}\right) \wedge \mathrm{d} c_{2} .
\end{aligned}
$$

Since $\xi_{1} \xi_{2}=1-z^{2}$ on $V$ Twhich differentiating gives

$$
\frac{\mathrm{d} \xi_{1}}{\xi_{1}}+\frac{\mathrm{d} \xi_{2}}{\xi_{2}}=-\frac{2 z}{1-z^{2}} \mathrm{~d} z .
$$

Whence

$$
\left.\omega\right|_{V}=\frac{1}{1-z^{2}} \mathrm{~d} z \wedge \mathrm{d} y+i\left(\frac{z}{z^{2}-1} \mathrm{~d} z-\frac{\mathrm{d} \xi_{1}}{\xi_{1}}\right) \wedge \mathrm{d} c_{2} .
$$

Differentiating $y^{2}=2\left(c_{1}-z\right)\left(1-z^{2}\right)-c_{2}^{2}$ gives

$$
\mathrm{d} y=\frac{\left(3 z^{2}-2 c_{1} z-1\right)}{y} \mathrm{~d} z+\frac{\left(1-z^{2}\right)}{y} \mathrm{~d} c_{1}-\frac{c_{2}}{y} \mathrm{~d} c_{2},
$$

which substituted into (12) yields (10).

We now prove

Proposition 4.2. The symplectic form $\omega$ can be extended holomorphically to $\bar{M}^{\text {ns }}$. 
Proof. We will use equation (10) of proposition 4.1. We must extend $\omega$ to points of $\bar{M}^{n s} \backslash M^{n s}$. Consider the rational functions $s=z / y$ and $t=1 / y$ on $E^{n s}$. These functions can be extended holomorphically to the point at infinity of each $E_{c}$. At infinity they take the values $s=0 \Gamma t=0 \Gamma$ where $s$ is the local parmeter at infinity. Also define new variables $\sigma_{i}=\xi_{i} / y$ and $\tau_{i}=\eta_{i} / y$ for $i=1,2$. In terms of these variables the defining equations of an affine part of $\bar{M}^{n s}$ read

$$
\begin{aligned}
\sigma_{1} \sigma_{2} & =t^{2}-s^{2} \\
\sigma_{1} \tau_{2} & =-s-i c_{2} t^{2} \\
\sigma_{2} \tau_{1} & =-s+i c_{2} t^{2} \\
\tau_{1} \tau_{2} & =-1+2\left(c_{1} t^{2}-s t\right) .
\end{aligned}
$$

This affine part includes the points of $\bar{M}^{n s} \backslash M^{n s}$. Moreover $\Gamma$ the defining equation for $E^{\text {ns }}$ becomes

$$
t=2\left(s^{2}-t^{2}\right)\left(s-c_{1} t\right)-c_{2}^{2} t^{3} .
$$

From the above equations we see that $\tau_{1} \tau_{2}=-1$ when $s=t=0$. In particular $\Gamma \tau_{1}$ and $\tau_{2}$ are nonzero above the points at infinity of $M^{n s}$. Hence $\sigma_{1}=\sigma_{2}=0$ there. Let us rewrite $\omega$ in terms of $s, t, \tau_{1}$ Tand $\tau_{2}$. A straightforward calculation gives

$$
\frac{\mathrm{d} z}{y}=\mathrm{d} s-\frac{s}{t} \mathrm{~d} t
$$

Differentiating (13) we obtain

$$
\mathrm{d} t=\frac{\left(6 s^{2}-2 c_{1} s t-2 t^{2}\right) \mathrm{d} s-2 t\left(s^{2}-t^{2}\right) \mathrm{d} c_{1}-2 c_{2} t^{3} \mathrm{~d} c_{2}}{1+4 t\left(s-c_{1} t\right)+2 c_{1}\left(s^{2}-t^{2}\right)+3 c_{2}^{2} t^{2}} .
$$

Thus $\frac{s}{t} \mathrm{~d} t$ is holomorphic at $s=t=0$ except possibly for the term $6 \frac{s^{3}}{t} \mathrm{~d} t$. HoweverTusing (13) we see that $s^{3}$ equals $t$ times a polynomial in $s$ and $t$. Thus $s^{3} / t$ is holomorphic at infinity and hence the 1 -form $\frac{\mathrm{d} z}{y}$ can be holomorphically extended to the points above $s=t=0$. A similar argument holds for the 2 -form $\frac{\mathrm{d} z}{y} \wedge \mathrm{d} c_{1}$.

Now let us turn to the part of $\omega$ with $\mathrm{d} c_{2}$. First note that $\frac{c_{2}}{z^{2}-1} \frac{\mathrm{d} z}{y}$ is holomorphic at infinity. Hence we can ignore that term. Next we rewrite the 1 -form $\frac{z y}{z^{2}-1} \frac{\mathrm{d} z}{y}-\frac{\mathrm{d} \xi_{1}}{\xi_{1}}$. Using the relation $\xi_{1}=-\left(z y+i c_{2}\right) / y \tau_{2}$ we obtain

$$
\frac{\mathrm{d} \tau_{2}}{\tau_{2}}+\frac{z \mathrm{~d} z}{z^{2}-1}+\frac{\mathrm{d} y}{y}-\frac{\mathrm{d}\left(z y+i c_{2}\right)}{z y+i c_{2}} .
$$


The 1 -form $\frac{\mathrm{d} \tau_{2}}{\tau_{2}}$ is holomorphic at infinity since $\tau_{2} \neq 0$ there. The last three terms are logarithmic derivatives which together equal

$$
\frac{1}{2} \frac{\mathrm{d}\left[\left(1-1 / z^{2}\right) /\left(1+i c_{2} / z y\right)\right]}{\left(1-1 / z^{2}\right) /\left(1+i c_{2} / z y\right)^{2}} .
$$

Since $\left(1-1 / z^{2}\right) /\left(1+i c_{2} / z y\right)^{2}$ is holomorphic at infinity derivative is also. Hence $\omega$ is holomorphic at infinity.

Corollary 4.3. Let $c=\left(c_{1}, c_{2}\right) \notin \Delta$. The differential forms

$$
\omega_{1}=\frac{\mathrm{d} z}{y}, \quad \omega_{2}=i\left(\frac{z y-i c_{2}}{z^{2}-1}\right) \frac{\mathrm{d} z}{y}-\frac{\mathrm{d} \xi_{1}}{\xi_{1}}
$$

restricted to the Zariski open subset of $M_{c}$ where $y\left(z^{2}-1\right) \neq 0$ can be extended holomorphically to all of $\bar{M}_{c}$. The extension of the 1-forms $\omega_{1}, \omega_{2}$ to $\bar{M}_{c}$ will again be denoted by $\omega_{1}, \omega_{2}$.

Proof. This follows from the observation that the 3 -forms $\frac{\mathrm{d} z}{y} \wedge$ $\mathrm{d} c_{1} \wedge \mathrm{d} c_{2}=\omega \wedge \mathrm{d} c_{2}$ and $\left(i \frac{z y-i c_{2}}{z^{2}-1} \frac{\mathrm{d} z}{y}-\frac{\mathrm{d} \xi_{1}}{\xi_{1}}\right) \wedge \mathrm{d} c_{2} \wedge \mathrm{d} c_{1}=\omega \wedge \mathrm{d} c_{1}$ are holomorphic on $\bar{M}^{n s}$.

Motivated by the form of $\omega$ in proposition 4.1 we construct the complex analogue of the period lattice on $\bar{M}_{c}$ as follows. Until further notice we assume that $c \notin \Delta$. Fix a base point $p_{0} \in \bar{M}_{c}$ and consider the Abel-Jacobi map

$$
\mathcal{A} \mathcal{J}: \bar{M}_{c} \rightarrow \mathbf{C}^{2}: p \rightarrow\left(u_{1}(p), u_{2}(p)\right)=\left(\int_{p_{0}}^{p} \omega_{1}, \int_{p_{0}}^{p} \omega_{2}\right),
$$

where the integrals are taken along a path $\gamma$ on $\bar{M}_{c}$ which joins $p_{0}$ to $p$. Since the 1 -forms $\omega_{1}$ and $\omega_{2}$ are closed $\Gamma$ the functions $u_{1}$ and $u_{2}$ depend only on the homology class of the path on $\bar{M}_{c}$ joining $p_{0}$ to $p$. The map $\mathcal{A} \mathcal{J}$ is multivalued due to the fact that $p_{0}$ and $p$ can be joined by nonhomologous paths $\Gamma$ say $\gamma$ and $\tilde{\gamma}$. The difference in the corresponding values of $\mathcal{A} \mathcal{J}$ is given by a vector

$$
\left(\int_{\Gamma} \omega_{1}, \int_{\Gamma} \omega_{2}\right)
$$

where $\Gamma$ is the closed path beginning and ending at $p_{0}$ which is formed by first tracing out $\gamma$ and then tracing out $\tilde{\gamma}$ backwards. Thus the multivaluedness of $\mathcal{A} \mathcal{J}$ is given by vectors of the form (15) where $\Gamma$ ranges over $\mathrm{H}_{1}\left(\bar{M}_{c}, \mathbf{Z}\right)$. Let

$$
L_{c}=\left\{\left(\int_{\Gamma} \omega_{1}, \int_{\Gamma} \omega_{2}\right) \in \mathbf{C}^{2} \mid \Gamma \in \mathrm{H}_{1}\left(\bar{M}_{c}, \mathbf{Z}\right)\right\} .
$$


Theorem 4.4. Let $\gamma_{1}, \gamma_{2}$ be a $\mathbf{Z}$-basis of $\mathrm{H}_{1}\left(\bar{E}_{c}, \mathbf{Z}\right)$. Suppose that the curves $\gamma_{i}$ avoid the points of $\bar{E}_{c}$ where $z^{2}=1$ or the point at infinity. For $j=1,2$ define

$$
\lambda_{j}=\int_{\gamma_{j}} \frac{\mathrm{d} z}{y}, \quad \mu_{j}=i \int_{\gamma_{j}} \frac{z y-i c_{2}}{z^{2}-1} \frac{\mathrm{d} z}{y} .
$$

Then $L_{c}$ is a lattice in $\mathbf{C}^{2}$ called the period lattice. $L_{c}$ has $\mathbf{Z}$-rank three and is generated by the vectors

$$
\left(\lambda_{1}, \mu_{1}\right), \quad\left(\lambda_{1}, \mu_{1}\right), \quad(0,2 \pi) .
$$

Proof. Let $U_{c}=\bar{E}_{c} \backslash\left\{z^{2}=1, \infty\right\}$. Note that the three points $\left(1,-i c_{2}\right) \Gamma\left(-1, i c_{2}\right)$ and infinity are precisely the poles of the differential form $\Omega=i \frac{z y-i c_{2}}{z^{2}-1} \frac{\mathrm{d} z}{y}$ on $\bar{E}_{c}$ which are all of first order. Since $\Omega=i\left(\frac{z y-i c_{2}}{y(z+1)}\right) \frac{\mathrm{d} z}{z-1} \Gamma$ the residue of the pole at $\left(1,-i c_{2}\right)$ is $-i$. Similarly the residue at $\left(-1, i c_{2}\right)$ is $-i$. Hence the residue at infinity is $2 i$. Consequently The value of $\int_{\gamma} \Omega \Gamma$ where $\gamma$ is a closed loop in $U_{c}$ Tis determined by the homology class of $\gamma \in \mathrm{H}_{1}\left(\bar{E}_{c}, \mathbf{Z}\right)$ up to an integer multiple of $2 \pi$.

Let $\Gamma$ be any closed path in $\bar{M}_{c}$. Without loss of generality we can assume that $\Gamma$ lies in the open subset $V_{c}=\bar{\pi}^{-1}\left(U_{c}\right)$. Since $z^{2} \neq 1, \infty$ in $V_{c}$ Tit follows that $\xi_{1} \neq 0$. Moreover $\Gamma$ the map

$$
V_{c} \rightarrow U_{c} \times \mathbf{C}^{*}:\left(\xi_{1}, \xi_{2}, z, \eta_{1}, \eta_{2}, y\right) \rightarrow\left(z, y, \xi_{1}\right)
$$

is an diffeomorphism. The image in $U_{c}$ of the curve $\Gamma$ under the projection $\pi$ is homologous to an integral linear combination of $\gamma_{1}$ and $\gamma_{2} \Gamma$ say $\bar{\pi}(\Gamma)=m_{1} \gamma_{1}+m_{2} \gamma_{2}$. Hence

$$
\int_{\Gamma} \omega_{1}=\int_{\bar{\pi}(\Gamma)} \frac{\mathrm{d} z}{y}=m_{1} \lambda_{1}+m_{2} \lambda_{2}
$$

and

$$
\begin{aligned}
\int_{\Gamma} \omega_{2} & =i \int_{\Gamma} \frac{\mathrm{d} \xi_{1}}{\xi_{1}}+i \int_{\bar{\pi}(\Gamma)} \frac{z y-i c_{2}}{z^{2}-1} \frac{\mathrm{d} z}{y} \\
& =i \int_{\Gamma} \frac{\mathrm{d} \xi_{1}}{\xi_{1}}+\left(m_{1} \mu_{1}+m_{2} \mu_{2}\right) \bmod 2 \pi
\end{aligned}
$$

Since we can let $\Gamma$ encircle $\xi_{1}=0$ as many times as we want $\Gamma$ the integral $i \int_{\Gamma} \frac{\mathrm{d} \xi_{1}}{\dot{\xi}_{1}}$ can be any multiple of $2 \pi$. Consequently $\Gamma$ the set of values of $\int_{\Gamma} \omega_{2} \Gamma$ where $\bar{\pi}(\Gamma)$ is homologous to $m_{1} \gamma_{1}+m_{2} \gamma_{2} \Gamma$ is $m_{1} \mu_{1}+m_{2} \mu_{2}+2 k \pi$ where $k \in \mathbf{Z}$ is arbitrary. This proves the theorem. 
Another way of stating the result of theorem 4.4 is that the vectors

$$
\left(\begin{array}{c}
0 \\
2 \pi
\end{array}\right), \quad\left(\begin{array}{l}
\int_{\bar{\pi}^{-1}\left(\gamma_{1}\right)} \omega_{1} \\
\int_{\bar{\pi}^{-1}\left(\gamma_{1}\right)} \omega_{2}
\end{array}\right), \quad\left(\begin{array}{l}
\int_{\bar{\pi}^{-1}\left(\gamma_{2}\right)} \omega_{1} \\
\int_{\bar{\pi}^{-1}\left(\gamma_{2}\right)} \omega_{2}
\end{array}\right)
$$

generate the period lattice $L_{c}$.

Because $\omega_{1} \wedge \omega_{2} \neq 0$ Tthe Abel-Jacobi map $\mathcal{A} \mathcal{J}$ is locally invertible. It will turn out that this local inverse can be extended to all of $\mathbf{C}^{2} \Gamma$ thus defining a global holomorphic inverse. Although we could prove this at this point $\Gamma$ we prefer to give an explicit construction of the inverse to $\mathcal{A} \mathcal{J}$ in the next section.

Having discussed the period lattice we now prove the following complex analogue of action angle coordinates.

Proposition 4.5. For each fixed $c \in \mathbf{C}^{2} \backslash \Delta$ there is an open neighborhood $U$ of $\bar{M}_{c}$ in $\bar{M}^{n s}$ and local coordinates $\left(c_{1}, c_{2}, \tilde{u}_{1}, \tilde{u}_{2}\right)$ on $U$ such that the symplectic form $\omega$ is $\mathrm{d} \tilde{u}_{1} \wedge \mathrm{d} c_{1}+\mathrm{d} \tilde{u}_{2} \wedge \mathrm{d} c_{2}$. The coordinates $\left(c_{1}, c_{2}, \tilde{u}_{1}, \tilde{u}_{2}\right)$ are called complex action-angle coordinates. In actionangle coordinates the vector fields $X_{H}$ and $X_{L}$ on $\bar{M}_{c}$ are $\frac{\partial}{\partial \widetilde{u}_{1}}$ and $\frac{\partial}{\partial \widetilde{u}_{2}}$, respectively.

Before proving the proposition we construct some preliminary local coordinates. To construct them we need a local section of the surjective submersion

$$
\bar{\sigma}: \bar{M}^{n s} \rightarrow \mathbf{C}^{2} \backslash \Delta:\left(\left(\xi_{1}: \xi_{2}: z: \eta_{1}: \eta_{2}: y: u\right),\left(c_{1}, c_{2}\right)\right) \rightarrow\left(c_{1}, c_{2}\right) .
$$

Note that the map $\bar{\sigma}$ is the composition the surjective submersion

$$
\bar{\pi}: \bar{M}^{n s} \rightarrow \bar{E}^{n s}
$$

with the surjective submersion

$$
\bar{\rho}: \bar{E}^{n s} \rightarrow \mathbf{C}^{2} \backslash \Delta:\left((z: y: u),\left(c_{1}, c_{2}\right)\right) \rightarrow\left(c_{1}, c_{2}\right) .
$$

Since the fiber $\bar{\rho}^{-1}\left(c_{1}, c_{2}\right)$ is $\bar{E}_{c}$ Twhich is compact $\Gamma \bar{\rho}$ is a locally trivial bundle. By construction the bundle $\bar{\pi}$ is locally trivial. Consequently $\Gamma$ $\bar{\sigma}$ is a locally trivial bundle. Fix $c \in \mathbf{C}^{2} \backslash \Delta$. Then $\bar{\sigma}^{-1}(c)=\bar{M}_{c}$. There is an open neighborhood $W$ of $c$ in $\mathbf{C}^{2} \backslash \Delta$ such that the bundle $\bar{\sigma} \mid U: U=\bar{\sigma}^{-1}(W) \rightarrow W$ is trivial. Let

$$
\tau: W \subseteq \mathbf{C}^{2} \backslash \Delta \rightarrow U=\bar{\sigma}^{-1}(W) \subseteq \bar{M}^{n s}
$$

be a section of $\bar{\sigma} \mid U$. We now prove 
LEMMA 4.6. The map

$$
\begin{aligned}
\psi: U & =\bar{\sigma}^{-1}(W) \subseteq \bar{M}^{n s} \rightarrow W \times \mathbf{C}^{2} \subseteq W \times\left(\mathbf{C}^{2} \backslash \Delta\right): \\
p & \rightarrow\left(\bar{\sigma}(p), \int_{\tau(\bar{\sigma}(p))}^{p} \omega_{1}, \int_{\tau(\bar{\sigma}(p))}^{p} \omega_{2}\right)=\left(c_{1}, c_{2}, \widehat{u}_{1}, \widehat{u}_{2}\right)
\end{aligned}
$$

is a local diffeomorphism.

Proof. Since $\operatorname{dim} \bar{\sigma}^{-1}(W)=\operatorname{dim}\left(W \times \mathbf{C}^{2}\right) \Gamma$ it suffices to show that $\psi$ is a submersion. Let $\pi_{1}, \pi_{2}, \pi_{3}$ be the projections onto the first $\Gamma$ second and third factors of $W \times(\mathbf{C} \times \mathbf{C})$. Clearly the map $\pi_{1} \circ \psi$ is a submersion. Now

$$
\mathrm{d}\left(\pi_{2} \circ \psi\right)(p)=\mathrm{d} \hat{u}_{1}(p)=\omega_{1}(p)-\tau^{*} \omega_{1}(\bar{\sigma}(p))
$$

and

$$
\mathrm{d}\left(\pi_{3} \circ \psi\right)(p)=\mathrm{d} \widehat{u}_{2}(p)=\omega_{2}(p)-\tau^{*} \omega_{2}(\bar{\sigma}(p)) .
$$

But $\mathrm{d}\left(\pi_{2} \circ \psi\right)(p) \wedge \mathrm{d}\left(\pi_{3} \circ \psi\right)(p)$ is nonzero because $\omega_{1} \wedge \omega_{2}=e_{1} \mathrm{~d} z \wedge \mathrm{d} \xi_{1}$ is nonzero and
a). $\omega_{1} \wedge \tau^{*} \omega_{2}=f_{1} \mathrm{~d} z \wedge \mathrm{d} c_{1}+f_{2} \mathrm{~d} z \wedge \mathrm{d} c_{2} \Gamma$
b). $\omega_{2} \wedge \tau^{*} \omega_{1}=g_{1} \mathrm{~d} z \wedge \mathrm{d} c_{1}+g_{2} \mathrm{~d} \xi_{1} \wedge \mathrm{d} c_{1}+g_{3} \mathrm{~d} z \wedge \mathrm{d} c_{2}+g_{4} \mathrm{~d} \xi_{1} \wedge \mathrm{d} c_{2} \Gamma$
c). $\tau^{*} \omega_{1} \wedge \tau^{*} \omega_{2}=h_{1} \mathrm{~d} c_{1} \wedge \mathrm{d} c_{2}$.

Therefore the map $\left(\pi_{2} \times \pi_{3}\right) \circ \psi$ is a submersion $\Gamma$ which implies that $\psi$ is a submersion.

Proof (of proposition 4.5). From lemma 4.6 it follows that $\left(c_{1}, c_{2}, \widehat{u}_{1}, \widehat{u}_{2}\right)$ are local coordinates on $U$. We now compute the 2 -form

$$
\begin{aligned}
& \mathrm{d} \hat{u}_{1} \wedge \mathrm{d} c_{1}+\mathrm{d} \hat{u}_{2} \wedge \mathrm{d} c_{2}=\left(\omega_{1}-\tau^{*} \omega_{1}\right) \wedge \mathrm{d} c_{1}+\left(\omega_{2}-\tau^{*} \omega_{2}\right) \wedge \mathrm{d} c_{2} \\
&= \omega_{1} \wedge \mathrm{d} c_{1}-h_{1}\left(c_{1}, c_{2}\right) \mathrm{d} c_{1} \wedge \mathrm{d} c_{2}+\omega_{2} \wedge \mathrm{d} c_{2}-h_{2}\left(c_{1}, c_{2}\right) \mathrm{d} c_{1} \wedge \mathrm{d} c_{2}, \\
& \text { since } \tau^{*} \omega_{1} \text { and } \tau^{*} \omega_{2} \text { are 1-forms on } W \times \mathrm{C}^{2} \\
&= \omega_{1} \wedge \mathrm{d} c_{1}+\omega_{2} \wedge \mathrm{d} c_{2}-h\left(c_{1}, c_{2}\right) \mathrm{d} c_{1} \wedge \mathrm{d} c_{2} .
\end{aligned}
$$

The 2-form $h\left(c_{1}, c_{2}\right) \mathrm{d} c_{1} \wedge \mathrm{d} c_{2}$ is a closed. Choosing the neighborhood $W$ to be simply connected and star shaped $\Gamma$ it follows that there is a 1 -form $\theta\left(c_{1}, c_{2}\right)=\theta_{1} \mathrm{~d} c_{1}+\theta_{2} \mathrm{~d} c_{2}$ such that

$$
-h\left(c_{1}, c_{2}\right) \mathrm{d} c_{1} \wedge \mathrm{d} c_{2}=\mathrm{d} \theta\left(c_{1}, c_{2}\right) .
$$

Adjust the local section $\tau$ of the trivial bundle $\bar{\sigma} \mid U$ so as to define new angle coordinates

$$
\tilde{u}_{1}=\widehat{u}_{1}-\theta_{1} \quad \tilde{u}_{2}=\widehat{u}_{2}-\theta_{2} .
$$


In action-angle coordinates $\left(c_{1}, c_{2}, \tilde{u}_{1}, \tilde{u}_{2}\right)$ we have

$$
\begin{aligned}
\mathrm{d} \tilde{u}_{1} \wedge \mathrm{d} c_{1}+\mathrm{d} \tilde{u}_{2} \wedge \mathrm{d} c_{2} & =\left(\mathrm{d} \widehat{u}_{1}-\mathrm{d} \theta_{1}\right) \wedge \mathrm{d} c_{1}+\left(\mathrm{d} \widehat{u}_{2}-\mathrm{d} \theta_{2}\right) \wedge \mathrm{d} c_{2} \\
& =\mathrm{d} \widehat{u}_{1} \wedge \mathrm{d} c_{1}+\mathrm{d} \widehat{u}_{2} \wedge \mathrm{d} c_{2}-\mathrm{d} \theta\left(c_{1}, c_{2}\right) \\
& =\omega_{1} \wedge \mathrm{d} c_{1}+\omega_{2} \wedge \mathrm{d} c_{2}
\end{aligned}
$$

using (21) and (20). But the right hand side of (22) is the symplectic form $\omega$ on $U=\bar{\sigma}^{-1}(W)$.

In action-angle coordinates on $U$ Tthe Hamiltonian function $H(6)$ is $c_{1}$. Thus

$$
\left.\mathrm{d} c_{1}=X_{H}\right\lrcorner\left(\mathrm{d} \tilde{u}_{1} \wedge \mathrm{d} c_{1}+\mathrm{d} \tilde{u}_{2} \wedge \mathrm{d} c_{2}\right),
$$

which implies that $X_{H}=\frac{\partial}{\partial \widetilde{u}_{1}}$. Similarly the momentum function $L$ (6) is $c_{2} \Gamma$ which implies that $X_{L}=\frac{\partial}{\partial \widetilde{u}_{2}}$. This completes the proof of proposition 4.5 .

An immediate consequence of proposition 4.5 is the fact that the complex spherical pendulum is Mumford-Jacobi completely integrable.

\section{The inverse of the Abel-Jacobi map}

In this section we show that the local inverse of the Abel-Jacobi map $\mathcal{A} \mathcal{J}$ (14) constructed in preceeding section extends to a global inverse defined on all of $\mathbf{C}^{2}$.

We use the same notation as in theorem 4.4. We now recall the basic properties of the Weierstrass elliptic functions $\wp \Gamma \zeta \Gamma$ and $\sigma$. For more details see [10Гchpt. XXГрp. 429-446]. Let $\Lambda_{c}$ be the lattice in $\mathrm{C}$ generated by the complex numbers $\lambda_{1}$ and $\lambda_{2}$ (16). Corresponding to $\Lambda_{c}$ there is the Weierstrass $\wp-$ function. There is also the $\zeta$-function which is characterized by the properties: 1). $\zeta^{\prime}(u)=-\wp(u)$ and 2$)$. $\zeta(u)=\frac{1}{u}+\mathrm{O}(u)$ about $u=0$. The $\zeta$ function is not periodic. In fact there are two complex numbers $\eta_{1}$ and $\eta_{2}$ Гcalled quasi-periods of $\bar{E}_{c} \Gamma$ such that

$$
\zeta\left(u+\lambda_{1}\right)=\zeta(u)+\eta_{1}, \quad \zeta\left(u+\lambda_{2}\right)=\zeta(u)+\eta_{2} .
$$

More generally corresponding to every $\lambda \in \Lambda_{c}$ there is a unique quasiperiod $\eta(\lambda)$ such that $\zeta(u+\lambda)=\zeta(u)+\eta(\lambda)$. When $\lambda=m_{1} \lambda_{1}+m_{2} \lambda_{2} \Gamma$ we see that $\eta(\lambda)=m_{1} \eta_{1}+m_{2} \eta_{2}$. Finally defined by

$$
\sigma(u)=u \prod_{\lambda \in \Lambda_{c}-\{0\}}\left(1-\frac{u}{\lambda}\right) \mathrm{e}^{u / \lambda+(u / \lambda)^{2} / 2},
$$


which satisfies $\sigma^{\prime}(u) / \sigma(u)=\zeta(u)$. Furthermore $\Gamma \sigma$ has the property that

$$
\sigma(u+\lambda)=\mathrm{e}^{\lambda / 2+\eta(\lambda) u} \sigma(u)
$$

for every $\lambda \in \Lambda_{c}$.

To construct the inverse of the Abel-Jacobi map $\mathcal{A} \mathcal{J}$ (14) we will need a product of $\sigma$-functions. Why the choices are made will become clear in the proof of theorem 5.1 below. Choose $a, b \in \mathbf{C}$ so that

$2 \wp(a)+\frac{1}{3} c_{1}=1, \quad 2 \wp^{\prime}(a)=-i c_{2}, \quad 2 \wp(b)+\frac{1}{3} c_{1}=-1, \quad 2 \wp^{\prime}(b)=i c_{2}$.

Then define the function

$$
\Sigma(u)=\mathrm{e}^{(\zeta(a)+\zeta(b)) u} \frac{\sigma(u-a) \sigma(u-b)}{\sigma(a) \sigma(b) \sigma(u)^{2}} .
$$

As a consequence of the properties of the $\sigma$-function we obtain

$$
\Sigma(u+\lambda)=\exp (-(a+b) \eta(\lambda)+(\zeta(a)+\zeta(b)) \lambda) \Sigma(u) .
$$

Moreover $\Gamma$ the principal part of $\Sigma$ at $u=0$ is $\frac{1}{u^{2}}$.

Theorem 5.1. For the point $p_{0} \in \bar{M}_{c}$ in the definition of the AbelJacobi map $\mathcal{A} \mathcal{J}$ (14) we take $\left(s, t, \sigma_{1}, \sigma_{2}, \tau_{1}, \tau_{2}\right)=(0,0,0,0,1,-1)$. Then the map $\mathcal{W}: \mathrm{C}^{2} \rightarrow \bar{M}_{c}:\left(u_{1}, u_{2}\right) \rightarrow\left(z, y, \xi_{1}\right)$, where

$$
\left(z, y, \xi_{1}\right)=\left(2 \wp\left(u_{1}\right)+\frac{1}{3} c_{1}, 2 \wp^{\prime}\left(u_{1}\right), \mathrm{e}^{i u_{2}} \Sigma\left(u_{1}\right)\right)
$$

is a global inverse of the Abel-Jacobi map. Since every fiber of $\mathcal{W}$ is the lattice $L_{c}$, it follows that $\bar{M}_{c}=\mathbf{C}^{2} / L_{c}$.

Proof. First we note that the inverse of the map

$$
\kappa: \bar{E}_{c} \rightarrow \mathbf{C}: \bar{\pi}(p) \rightarrow u_{1}(\bar{\pi}(p))=\int_{\bar{\pi}\left(p_{0}\right)}^{\bar{\pi}(p)} \omega_{1}=\int_{\bar{\pi}\left(p_{0}\right)}^{\bar{\pi}(p)} \frac{\mathrm{d} z}{y}
$$

is given by

$$
\rho: \mathbf{C} \rightarrow \bar{E}_{c}: u_{1} \rightarrow\left(2 \wp\left(u_{1}\right)+\frac{1}{3} c_{1}, 2 \wp^{\prime}\left(u_{1}\right)\right)=(z, y) .
$$

To see this replace the variables $z$ and $y$ in $\omega_{1}$ by their Weierstrass parametrizations. Then $\mathrm{d} u_{1}=\frac{\mathrm{d} z}{y}$ and $\rho(0)=\infty \in \bar{E}_{c}$.

We now consider $\int_{p_{0}}^{p} \omega_{2}$. First we replace the variables $z, y$ in $\omega_{2}$ by their Weierstrass parametrizations. Then $\frac{z y-i c_{2}}{z^{2}-1}$ becomes an elliptic function on $\bar{E}_{c}$ with poles only at $a+\Lambda_{c} \Gamma b+\Lambda_{c}$ and $0+\Lambda_{c} \Gamma$ which are of first order with residues $1 \Gamma 1$ Tand -2 respectively. Observe that the elliptic function

$$
Z\left(u_{1}\right)=\zeta\left(u_{1}-a\right)+\zeta\left(u_{1}-b\right)-2 \zeta\left(u_{1}\right)
$$

on $\bar{E}_{c}$ has the same poles and residues as $\frac{z y-i c_{2}}{z^{2}-1}$. Thus the difference of $\frac{z y-i c_{2}}{z^{2}-1}-Z\left(u_{1}\right)$ is a holomorphic elliptic function and hence is a bounded 
entire function on $\mathrm{C} \Gamma$ which is constant by Liouville's theorem. To compute this constant we evaluate this difference at $u_{1}=0$. We note that $\frac{z y-i c_{2}}{z^{2}-1}=-\frac{2}{u_{1}}+\mathrm{O}\left(u_{1}\right)$ and $Z\left(u_{1}\right)=-\frac{2}{u_{1}}-\zeta(a)-\zeta(b)+\mathrm{O}\left(u_{1}\right)$. Thus the constant is $\zeta(a)+\zeta(b) \Gamma$ that is $\Gamma$

$$
\frac{z y-i c_{2}}{z^{2}-1}=\zeta\left(u_{1}-a\right)+\zeta\left(u_{1}-b\right)-2 \zeta\left(u_{1}\right)+\zeta(a)+\zeta(b)=\frac{\Sigma^{\prime}\left(u_{1}\right)}{\Sigma\left(u_{1}\right)} .
$$

The second equality in the equation above explains why the function $\Sigma$ was constructed. For the moment let $p_{1}$ be a point in $\bar{M}_{c}$. Then

$$
\begin{aligned}
u_{2} & =\int_{p_{1}}^{p} \omega_{2}=i \int_{p_{1}}^{p} \frac{z\left(u_{1}\right) y\left(u_{1}\right)-i c_{2}}{z^{2}\left(u_{1}\right)-1} \mathrm{~d} u_{1}-i \int_{p_{1}}^{p} \frac{\mathrm{d} \xi_{1}}{\xi_{1}} \\
& =i \int_{p_{1}}^{p} \frac{\Sigma^{\prime}\left(u_{1}\right)}{\Sigma\left(u_{1}\right)} \mathrm{d} u_{1}-i \int_{p_{1}}^{p} \frac{\mathrm{d} \xi_{1}}{\xi_{1}} \\
& =i \log \left(\frac{\Sigma\left(u_{1}\right)}{\Sigma\left(u_{1}\left(p_{1}\right)\right)}\right)-i \log \left(\frac{\xi_{1}}{\xi\left(p_{1}\right)}\right) \\
& =i \log \left(\Sigma\left(u_{1}\right)\right)-i \log \xi_{1}+i \log \left(\frac{\xi\left(p_{1}\right)}{\Sigma\left(u_{1}\left(p_{1}\right)\right)}\right) .
\end{aligned}
$$

We now let $p_{1}$ converge to the point $p_{0}$ given in the statement of the theorem. Recall that $\Sigma\left(u_{1}\right)$ has principal part $\frac{1}{u_{1}^{2}}$ at $u=0$. Observe that

$$
\xi_{1}=-\frac{z y+i c_{2}}{y \tau_{2}}=-\frac{1}{\tau_{2}}\left(\frac{1}{u_{1}^{2}}+\cdots\right),
$$

using (8) and the fact that $y \tau_{2}=\eta_{2}$. Since $\tau_{2}$ converges to -1 as $p_{1}$ converges to $p_{0} \Gamma$ we see that $\lim _{p_{1} \rightarrow p_{0}}\left(\frac{\xi\left(p_{1}\right)}{\Sigma\left(u_{1}\left(p_{1}\right)\right)}\right)=1$. Hence

$$
u_{2}=i \log \Sigma\left(u_{1}\right)-i \log \xi_{1},
$$

which implies that $\xi_{1}=\mathrm{e}^{i u_{2}} \Sigma\left(u_{1}\right)$.

Since $\left(\xi_{1}, z, y\right)$ are coordinates for points $p$ in a Zariski open subset $V_{c}$ of $\bar{M}_{c}$ (see $\left.(17)\right)$ and $(\mathcal{W} \circ \mathcal{A} \mathcal{J})(p)=p$ एit follows that this equality holds for all points in $\bar{M}_{c}$. Thus $\mathcal{W}(23)$ is a global inverse of the Abel-Jacobi map $\mathcal{A} \mathcal{J}$.

In order to show that each fiber of $\mathcal{W}$ is the lattice $L_{c} \Gamma$ we first compute an explicit expression for the period

$$
\mu_{j}=i \int_{\gamma_{j}} \frac{z y-i c_{2}}{z^{2}-1} \frac{\mathrm{d} z}{y}
$$

see (16). Using the Weierstrass parametrization of $\bar{E}_{c}$ and choosing the curve

$$
[0,1] \rightarrow \mathbf{C}: t \rightarrow t \lambda_{j}+(1-t) u_{1}^{0}, \quad u_{1}^{0} \neq 0, a, b+\Lambda_{c}
$$


whose image under the map $\rho(24)$ is $\gamma_{j}$ Twe find that

$$
\begin{aligned}
\mu_{j} & =i \int_{u_{1}^{0}}^{u_{1}^{0}+\lambda_{j}} \frac{z\left(u_{1}\right) y\left(u_{1}\right)-i c_{2}}{z^{2}\left(u_{1}\right)-1} \mathrm{~d} u_{1}=i \int_{u_{1}^{0}}^{u_{1}^{0}+\lambda_{j}} \mathrm{~d} \log \Sigma\left(u_{1}\right) \\
& =i \log \left(\frac{\Sigma\left(u_{1}^{0}+\lambda_{j}\right)}{\Sigma\left(u_{1}^{0}\right)}\right)=-i\left[(a+b) \eta_{j}-(\zeta(a)+\zeta(b)) \lambda_{j}\right] .
\end{aligned}
$$

In other words $\Gamma$

$$
\mu(\lambda)=-i[(a+b) \eta(\lambda)-(\zeta(a)+\zeta(b)) \lambda] .
$$

Now suppose that $\left(u_{1}, u_{2}\right),\left(u_{1}^{\prime}, u_{2}^{\prime}\right) \in \mathcal{W}^{-1}\left(z, y, \xi_{1}\right)$. Then $u_{1}$ and $u_{1}^{\prime}$ correspond to the point $(z, y)=\left(2 \wp\left(u_{1}\right)+\frac{1}{3} c_{1}, 2 \wp^{\prime}\left(u_{1}\right)\right)$ on $\bar{E}_{c}$. Hence there is a $\lambda \in \Lambda_{c}$ such that $u_{1}^{\prime}=u_{1}+\lambda$. Since $u_{2}$ and $u_{2}^{\prime}$ correspond to

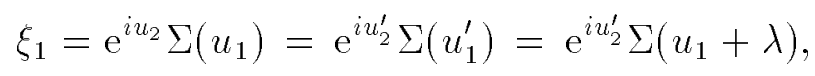

it follows that

$$
\begin{aligned}
u_{2}-u_{2}^{\prime} & =-i \log \left(\frac{\Sigma\left(u_{1}+\lambda\right)}{\Sigma\left(u_{1}\right)}\right) \\
& =i[(a+b) \eta(\lambda)-(\zeta(a)+\zeta(b)) \lambda]+2 \pi i k, \quad \text { for some } k \in \mathbf{Z} \\
& =-\mu(\lambda)+2 \pi i k .
\end{aligned}
$$

Thus $\left(u_{1}, u_{2}\right)$ and $\left(u_{1}^{\prime}, u_{2}^{\prime}\right)$ differ by some element of $L_{c}$. Consequently $\Gamma$ each fiber of $\mathcal{W}$ is the lattice $L_{c}$ Twhich shows that $\bar{M}_{c}$ is holomorphically diffeomorphic to $\mathrm{C}^{2} / L_{c}$.

\section{Monodromy}

In this section we show that the complexified spherical pendulum has monodromy. More precisely $\Gamma$ we show that there is a noncontractible loop $\Gamma$ in $\mathbf{C}^{2} \backslash \Delta$ such that the bundle of period lattices

$$
\nu: \coprod_{c \in \gamma} L_{c} \rightarrow \Gamma
$$

over $\Gamma$ has classifying map given by $\left(\begin{array}{rrr}1 & -1 & 0 \\ 0 & 1 & 0 \\ 0 & 0 & 1\end{array}\right)$.

For the moment $\Gamma$ consider the classical spherical pendulum. Let $c$ lie in the set of regular values $\mathcal{R}$ of its energy momentum mapping em. Then the motion of the spherical pendulum takes place on a smooth 2-dimensional torus $T_{c}^{2}$ in phase space $T S^{2}$. The image of $T_{c}^{2}$ under the tangent bundle projection map is an annular region $\mathcal{A}$ on $S^{2}$ bounded by two small circles lying in planes parallel to the equatorial plane. There are two important quantities associated to the motion on $T_{c}^{2}$ : one is the time $T(c)$ it takes starting on the upper boundary of $\mathcal{A}$ to 
the motion to return to this boundary for the first time and the other is the angle $\Theta(c)$ measured along the upper boundary from starting point to first return point. As is well known

$$
T(c)=2 \int_{z_{0}}^{z_{1}} \frac{\mathrm{d} z}{y} \text { and } \Theta(c)=2 \int_{z_{0}}^{z_{1}} \frac{c_{2}}{1-z^{2}} \frac{\mathrm{d} z}{y},
$$

where $y^{2}=f_{c}(z)=2\left(c_{1}-z\right)\left(1-z^{2}\right)-c_{2}^{2}$ and the discriminant of $f_{c}$ is positive. Here $-1<z_{0}<z_{1}<1<z_{1}$ are the three distinct real roots of $f_{c}$. Observe that $T(c)$ is the period of a holomorphic 1-form on $\bar{E}_{c}$ and $\Theta(c)$ is a period of a differential form of the third kind on $\bar{E}_{c}$. In $[5] \Gamma[4 \Gamma$ chpt. IV] it was shown that the real analytic function $\Theta$ on $\mathcal{R}$ is multivalued - decreasing by $2 \pi$ along any positively oriented closed curve $\Gamma$ in $\mathcal{R}$ which encircles the point $(1,0)$. Another way to say this is to use the real period lattice $\mathcal{L}_{c}$ defined by the 2-torus $T_{c}^{2}$. The lattice $\mathcal{L}_{c}$ is spanned by the vectors

$$
X_{L}(p) \text { and } T(e m(p)) \cdot X_{H}(p)+\frac{\Theta(e m(p))}{2 \pi} \cdot X_{L}(p),
$$

where $p \in T^{2}(c)$ and $X_{H}$ and $X_{L}$ are the Hamiltonian vector fields corresponding to the energy and angular momentum of the spherical pendulum. Then the bundle of real period lattices

$$
\tilde{\nu}: \coprod_{c \in \Gamma} \mathcal{L}_{c} \rightarrow \Gamma
$$

over $\Gamma$ has classifying map $\left(\begin{array}{rr}1 & -1 \\ 0 & 1\end{array}\right)$.

The next lemma describes the relation between the differential form $\Theta$ of the third kind on $\bar{E}_{c}$ and the holomorphic 1-form $\omega_{2}$ on $\bar{M}_{c}$.

LeMma 6.1. The period $\int_{\gamma} \omega_{2}$ modulo $\pi$ is minus the period $\int_{\bar{\pi} \circ \gamma} \Theta$ for any closed loop $\gamma$ on $\bar{M}_{c}$ which avoids the points with $z^{2}=1$.

Proof. First note that

$$
\int_{\gamma} \Theta=\int_{\gamma} \frac{c_{2}}{1-z^{2}} \frac{\mathrm{d} z}{y}=-i \int_{\gamma} \frac{\left(z y-i c_{2}\right)}{z^{2}-1} \frac{\mathrm{d} z}{y}+i \int_{\gamma} \frac{z y}{z^{2}-1} \frac{\mathrm{d} z}{y} .
$$

By theorem 4.4 the periods of $i \frac{z y-i c_{2}}{\left(z^{2}-1\right) y} \frac{\mathrm{d} z}{y}$ Ttaken over $\bar{E}_{c} \Gamma$ are $\Gamma$ modulo $2 \pi$ Tequal to the periods of the form $\omega_{2}$. The periods of $i \frac{z y}{z^{2}-1} \frac{\mathrm{d} z}{y}$ are multiples of $\pi \Gamma$ since this 1 -form is equal to $\frac{i}{2}$ times the logarithmic derivative $\frac{\mathrm{d}\left(z^{2}-1\right)}{z^{2}-1}$.

The main goal of this section is to prove the following 
Theorem 6.2. Consider the periods of the differential forms $\omega_{1}=$ $\frac{\mathrm{d} z}{y}$ and $\Theta=\frac{c_{2}}{1-z^{2}} \frac{\mathrm{d} z}{y}$ on $\bar{E}_{c}$ as analytic functions of $c=\left(c_{1}, c_{2}\right)$. Then there is a closed path $\Gamma$ in the c-space $\mathbf{C}^{2} \backslash \Delta$ such that after analytic continuation along one circuit of $\Gamma$, the periods of $\omega_{1}$ do not change and at least one period of $\Theta$ changes by $2 \pi$.

The idea of the proof is that we take a point $c_{0}$ in $c$-space close to the locus $\Delta$. Through $c_{0}$ we consider the complex curve $C$ of all parameter values $c$ such that $\bar{E}_{c}$ is isomorphic to $\bar{E}_{c_{0}}$. For every $c \in C$ we normalise the curve $E_{c}$ to a single curve $E$ in the Legendre form $v^{2}=2 u(u-1)(u-t) \Gamma$ where $t$ is a fixed large number. However $\Gamma$ the poles of the 1-form $\Theta$ still depend on the choice of $c \in C$. We let one of these poles $\Gamma$ called $s$ rrun over a path which encloses the points 0,1 in $\mathbf{C}$. Then one of the periods of $\Theta$ changes by $2 \pi$.

To carry out the proof change of variables as described in

Lemma 6.3. Suppose $s, t \in \mathbf{C}$ satisfy $|s|<2$ and $|t|>R$, where $R>0$ is sufficiently large $(R=100$ will $d o)$.

1. Define $a \in C$ by

$$
a=-\frac{1}{4}\left(-1-t+3 s-\sqrt{t^{2}+2(s-1)(t-1-3 s)}\right),
$$

where the square root is chosen so that it lies close to $t$. Then a is lies in $-\frac{t}{2}+\mathrm{C}\left[\left[s, \frac{1}{t}\right]\right]$ and the power series converges when $|s|<2,|t|>R$. 2. The substitution

$$
z=1+(u-s) / a, y=v / a^{3 / 2}
$$

transforms the defining equation $y^{2}=f_{c}(z)$ of $E_{c}$ into the defining equation $v^{2}=2 u(u-1)(u-t)$ of $E$.

3.

$$
c_{1}=(t-3 s+3 a+1) / a, \quad-c_{2}^{2}=2 s(s-1)(s-t) / a^{3} .
$$

4. The points on $E_{c}$ with $z=1$ correspond to points on $E$ with $u=s$, while the points with $z=-1$ correspond to those on $E$ with $u=-2 a+s$.

Proof (of lemma 6.3). 1. The statement about the analytic nature and the region of convergence of $a$ as function of $s, t$ is an elementary exercise.

2. We apply the inverse substitution $u=a(z-1)+s, v=a^{3 / 2} y$ to the curve $v^{2}=2 u(u-1)(u-t)$. We get

$$
y^{2}=2\left(z^{3}+b_{2} z^{2}+b_{1} z+b_{0}\right),
$$


where

$$
\begin{aligned}
& b_{0}=t \\
& b_{1}=\left(3 s^{2}+42 a t+t-4 s+3 a^{2}+2 a-6 a s-2 s t\right) / a^{2}, \\
& b_{2}=(-t+3 s-3 a-1) / a .
\end{aligned}
$$

The choice of $a$ made in equation (26) implies that $b_{1}=-1$. Therefore our transformed equation (28) reads

$$
y^{2}=2\left(z^{2}-1\right)\left(z+b_{2}\right)+2\left(1+b_{2}+b_{1}+b_{0}\right) .
$$

3. We can now identify $c_{1}$ with $-b_{2}$ and $-c_{2}^{2}$ with $2\left(1+b_{0}+b_{1}+b_{2}\right)$. A calculation shows that $-c_{2}^{2}=2 s(s-1)(s-t) / a^{3}$.

4. From $u=a(z-1)+s$ we easily see that $z= \pm 1$ implies that $u=s$ and $u=-2 a+s$ respectively.

LEMMA 6.4. Using the transformation (27), the 1-forms $\frac{\mathrm{d} z}{y}$ and $\Theta$ can be rewritten as

$$
\frac{\mathrm{d} z}{y}=a^{1 / 2} \frac{\mathrm{d} u}{v}, \quad \Theta=\frac{v(s)}{2 i}\left(\frac{1}{u-s}-\frac{1}{u+2 a-s}\right) \frac{\mathrm{d} u}{v} .
$$

Here $v(s)$ denotes a suitable choice of branch of the function

$$
\sqrt{2 s(s-1)(s-t)} \text {. }
$$

Proof. The transformation of $\frac{\mathrm{d} z}{y}$ is immediate. For $\Theta$ we note that

$$
\frac{c_{2}}{1-z^{2}} \frac{\mathrm{d} z}{y}=\frac{y(z)}{2 i}\left(\frac{1}{z+1}-\frac{1}{z-1}\right) \frac{\mathrm{d} z}{y},
$$

where $y(z)=\sqrt{2 f_{c}(z)}$. Carrying out the transformation (27) is now straightforward.

From the lemma 6.3 and 6.4 we see that it suffices to study the variation of the periods of the 1 -forms in (29).

We now assume that $t$ has a fixed value and we let $s$ run over a closed path $\Gamma$ which encircles the points 0,1 exactly once. Since $a(26)$ is close to $-\frac{t}{2}$ and is analytic in $s$ The value of $a^{1 / 2}$ returns to its original value after letting $s$ trace out $\Gamma$ once. Therefore the periods of $a^{1 / 2} \frac{\mathrm{d} u}{v}$ return to their original values. It remains to study the variation of the periods of $\Theta$. For this we need the additional 
Lemma 6.5. Consider the elliptic curve E defined by

$$
v^{2}=g(u)=2 u(u-1)(u-t) .
$$

Let $s$ be a complex number $\neq 0,1, t$ and let $\gamma$ be a closed path on $E$ avoiding the points where $u=s$. Define

$$
p(s)=\int_{\gamma} \frac{v(s)}{u-s} \frac{\mathrm{d} u}{v} .
$$

Then

$$
p^{\prime}(s)=\frac{s}{v(s)} \int_{\gamma} \frac{\mathrm{d} u}{v}-\frac{1}{v(s)} \int_{\gamma} u \frac{\mathrm{d} u}{v},
$$

where' denotes differentiation with respect to $s$.

Proof. Differentating $p$ under the integral sign gives

$$
p^{\prime}(s)=\int_{\gamma} \frac{\mathrm{d}}{\mathrm{d} s}\left(\frac{v(s)}{u-s}\right) \frac{\mathrm{d} u}{v} .
$$

But

$$
\frac{\mathrm{d}}{\mathrm{d} s}\left(\frac{v(s)}{u-s}\right)=\frac{v^{\prime}(s)}{u-s}+\frac{v(s)}{(u-s)^{2}}=\left(\frac{1}{2} \frac{g^{\prime}(s)}{u-s}+\frac{g(s)}{(u-s)^{2}}\right) \frac{1}{v(s)},
$$

using $v(s)^{2}=g(s)$ and $2 v(s) v^{\prime}(s)=g^{\prime}(s)$. Hence

$$
p^{\prime}(s)=\frac{1}{v(s)} \int_{\gamma}\left(\frac{1}{2} \frac{g^{\prime}(s)}{u-s}+\frac{g(s)}{(u-s)^{2}}\right) \frac{\mathrm{d} u}{v} .
$$

A straightforward computation $\Gamma$ using the fact that $g(u)$ is a cubic polynomial with leading coefficient 2 Tgives

$$
\mathrm{d}\left(\frac{v}{u-s}\right)=\frac{1}{2} \frac{g^{\prime}(s)}{u-s} \frac{\mathrm{d} u}{v}+\frac{g(s)}{(u-s)^{2}} \frac{\mathrm{d} u}{v}+(u-s) \frac{\mathrm{d} u}{v} .
$$

Hence

$$
p^{\prime}(s)=\frac{1}{v(s)}\left[\int_{\gamma} \mathrm{d}\left(\frac{v}{u-s}\right)-\int_{\gamma}(u-s) \frac{\mathrm{d} u}{v}\right] .
$$

Since a period of an exact form is zero we obtain (30).

Proof (of theorem 6.2). We use the same notation as in the discussion above. The variation $\Delta_{\Gamma} p$ of $p(s)$ along the path $\Gamma$ is given by $\int_{\Gamma} p^{\prime}(s) \mathrm{d} s$. Hence $\mathrm{bby}$ lemma 6.5

$$
\Delta_{\Gamma} p=\int_{\Gamma} \frac{s \mathrm{~d} s}{v(s)} \int_{\gamma} \frac{\mathrm{d} u}{v}-\int_{\Gamma} \frac{\mathrm{d} s}{v(s)} \int_{\gamma} \frac{u \mathrm{~d} u}{v} .
$$

The Weierstrass parametrisation of the curve $E$ is given by

$$
u=2 \wp(\tau)-\frac{1}{3}(t+1), \quad v=2 \wp^{\prime}(\tau) .
$$


Let $\tau_{\gamma}, \tau_{\Gamma}$ be the elements in the lattice $L$ such that $\bar{E}=\mathrm{C} / L$ which correspond to the curves $\gamma$ and $\Gamma$. Let $\eta_{\gamma}$ and $\eta_{\Gamma}$ be the quasi-periods of the Weierstrass $\zeta$-function for $L$. Then

$$
\Delta_{\Gamma} p=-2\left(\eta_{\Gamma} \tau_{\gamma}-\eta_{\gamma} \tau_{\Gamma}\right) .
$$

From the Legendre relations for elliptic functions it follows that

$$
\Delta_{\Gamma} p=4 n \pi i,
$$

where $n$ the negative of the intersection number of $\Gamma$ and $\gamma$. By choosing $\gamma$ such that it intersects $\Gamma$ exactly once with intersection number $1 \Gamma$ we can see to it that $p(s)$ changes by $4 \pi i$ as $s$ runs over $\Gamma$ once.

To prove the theorem we must show that $p(-2 a+s)$ does not change value if we let $s$ runs over $\Gamma$. To that end we write $s^{\prime}=-2 a+s$ and note that it depends on $s, t$ via $s^{\prime}=t+\frac{s-s^{2}}{t}+O\left(\frac{1}{t^{2}}\right)$. Since $s-s^{2}$ encircles the origin twice if we let $s$ run over $\Gamma$ Twe see that $s^{\prime}$ encircles the point $t$ twice. Denote this path by $\Gamma^{\prime}$. Since $\Gamma^{\prime}$ lifts to a closed path in the $\tau$-plane $\Gamma$ we get $\Delta_{\Gamma^{\prime}} p=0$.

In lemma 6.4 we saw that

$$
\Theta=\frac{1}{2 i}\left(\frac{v(s)}{u-s} \frac{\mathrm{d} u}{v}-\frac{v(s-2 a)}{u+2 a-s}\right) \frac{\mathrm{d} u}{v} .
$$

Thus we conclude that there is a least one period of $\Theta$ which changes by $\frac{1}{2 i} 4 \pi i=2 \pi$ when we make a circuit of $\Gamma$.

We are now in a position to prove

Corollary 6.6 (to theorem 6.2). Running over the curve $\Gamma$ once, the period of $\omega_{1}$ does not change, whereas one of the periods of $\omega_{2}$ decreases by $2 \pi$.

Proof. This follows immediately from lemma 6.1.

Another way to state the result of corollary 6.6 is that after making a circuit of $Г \Gamma$ the basis

$$
\left(\begin{array}{c}
0 \\
2 \pi
\end{array}\right), \quad\left(\begin{array}{l}
\int_{\bar{\pi}^{-1}\left(\gamma_{1}\right)} \omega_{1} \\
\int_{\bar{\pi}^{-1}\left(\gamma_{1}\right)} \omega_{2}
\end{array}\right), \quad\left(\begin{array}{l}
\int_{\bar{\pi}^{-1}\left(\gamma_{2}\right)} \omega_{1} \\
\int_{\bar{\pi}^{-1}\left(\gamma_{2}\right)} \omega_{2}
\end{array}\right)
$$

of the period lattice $L_{c_{0}}$ for $c_{0} \in \Gamma$ becomes either the basis

$$
\left(\begin{array}{c}
0 \\
2 \pi
\end{array}\right), \quad\left(\begin{array}{c}
\int_{\bar{\pi}^{-1}\left(\gamma_{1}\right)} \omega_{1} \\
\int_{\bar{\pi}^{-1}\left(\gamma_{1}\right)} \omega_{2}-2 \pi
\end{array}\right), \quad\left(\begin{array}{l}
\int_{\bar{\pi}^{-1}\left(\gamma_{2}\right)} \omega_{1} \\
\int_{\bar{\pi}^{-1}\left(\gamma_{2}\right)} \omega_{2}
\end{array}\right),
$$


in which case the bundle of period lattices over $\Gamma$ has classifying map $M_{1}=\left(\begin{array}{rrr}1 & -1 & 0 \\ 0 & 1 & 0 \\ 0 & 0 & 1\end{array}\right)$ or the basis

$$
\left(\begin{array}{c}
0 \\
2 \pi
\end{array}\right), \quad\left(\begin{array}{l}
\int_{\bar{\pi}^{-1}\left(\gamma_{1}\right)} \omega_{1} \\
\int_{\bar{\pi}^{-1}\left(\gamma_{1}\right)} \omega_{2}
\end{array}\right), \quad\left(\begin{array}{c}
\int_{\bar{\pi}^{-1}\left(\gamma_{2}\right)} \omega_{1} \\
\int_{\bar{\pi}^{-1}\left(\gamma_{2}\right)} \omega_{2}-2 \pi
\end{array}\right)
$$

for which the classifying map is $M_{2}=\left(\begin{array}{ccc}1 & 0 & -1 \\ 0 & 1 & 0 \\ 0 & 0 & 1\end{array}\right)$. Since

$$
\left(\begin{array}{rrr}
-1 & 0 & 0 \\
0 & 0 & -1 \\
0 & -1 & 0
\end{array}\right) M_{2}\left(\begin{array}{rrr}
-1 & 0 & 0 \\
0 & 0 & -1 \\
0 & -1 & 0
\end{array}\right)=M_{1},
$$

the period lattice bundles over $\Gamma$ with classifying maps $M_{1}$ and $M_{2}$ are isomorphic. This completes our treatment of monodromy in the complex spherical pendulum.

\section{References}

[1] Alber, S. and Marsden, J.E., Semiclassical monodromy and the spherical pendulum as a complex Hamiltonian system, Fields Institute Communications, $\mathbf{8}$ (1996), 1-18.

[2] M. Audin, Monodromy in the spherical pendulum, preprint September 2000, IRMA, Strasbourg.

[3] Brieskorn, E. and Knörrer, H., Plane Algebraic Curves, Birkhäuser, Boston, 1986.

[4] Cushman, R.H. and Bates, L.M., Global Aspects of Classical Integrable Systems, Birkhäuser, Basel, 1997.

[5] Duistermaat, J.J, On global action angle coordinates, Commun. Pure Appl. Math. 33 (1980) 687-706.

[6] L. Gavrilov and O. Vivolo, The real period function of the $A_{3}$ singularity and perturbations of the spherical pendulum, Compositio Mathematica, 123 (2000) $167-184$.

[7] Griffiths, P. and Harris, J., Principles of Algebraic Geometry, Wiley, N.Y., 1978.

[8] Knörrer, H., Letter to R. Cushman dated 20 July 1983.

[9] Mumford, D., Tata Lectures on Theta II, Birkhäuser, Boston, 1984.

[10] Whittaker, E.T. and Watson, G.N., A Course on Modern Analysis, $4^{\text {th }}$ ed., Cambridge University Press, Cambridge, UK., 1980.

Mathematics Institute, University of Utrecht, 3508Ta Utrecht, The Netherlands

Mathematics Institute, University of Utrecht, 3508TA Utrecht, THE Netherlands 